\title{
Modelo en Series de Tiempo del Retardo del Cambio de Canal en una Transmisión IPTV
}

\author{
Ricardo Ferro, José Rodríguez, Cesar Hernández* \\ Universidad Distrital Francisco José de Caldas, Facultad de Ingeniería y Tecnológica. Carrera 7 No. $40 \mathrm{~B}$ \\ 53. Bogotá DC, Colombia. (e-mail: rifer2411@gmail.com, jirodriguezm@udistrital.edu.co, \\ cahernandezs@udistrital.edu.co) \\ * autor a quien debe ser dirigida la correspondencia
}

Recibido Jul. 20, 2015; Aceptado Sep. 10, 2015; Versión final Oct. 3, 2015, Publicado Dic. 2015

\section{Resumen}

Se presenta el análisis y modelado del comportamiento del retardo generado por una red IPTV a través de series de tiempo, en el momento de realizar una solicitud de cambio de canal. En el estudio se toma en cuenta cada una de las etapas donde el servicio pudiera estar disponible, reduciendo el error promedio del retardo. A través del diseño y simulación de una red IPTV, se desarrolla el análisis del retardo en el cambio de canal para ocho escenarios, desde el usuario hasta la cabecera, considerando el nivel de congestión de la red. La validación de los modelos propuestos se realiza con datos de retardo reales capturados en una red IPTV. Los resultados muestran que cuando la red esta congestionada el modelo que mejor se adapta es el autorregresivo y en caso contrario el de promedios móviles.

Palabras clave: calidad de servicio; cambio de canal; IPTV; modelado; retardo

\section{Time Series Model for Channel Zapping Delay in an IPTV Transmission}

\begin{abstract}
The analysis and modeling of the delay behavior for channel change request in an IPTV network, through time series are presented. The study takes into account each of the stages where the service would be available, reducing the average delay error. Through the design and simulation of an IPTV network, the delay analysis is developed for eight scenarios, from the user to the header, considering the level of network's congestion. The validation of the proposed models is done with real delay data captured in an IPTV network. The results show that when the network is congested the model that best fits the data is the autoregressive and otherwise is the moving average.
\end{abstract}

Keywords: delay; channel zapping; IPTV; modeling; quality of service 


\section{INTRODUCCIÓN}

IPTV es un sistema completo mediante el cual la señal de televisión es entregada a los usuarios sobre el protocolo IP (Internet Protocol). Este sistema está formado por los servidores del contenido, encargados de codificar la señal y fragmentarla, encapsulando los paquetes para ofrecerlos en la red IP core, mediante Multicast o unicast. Una de las ventajas que tiene los sistemas de TV sobre IP es la gestión que se ejerce sobre el tráfico para garantizar niveles de calidad de servicio (QoS) más elevados. Donde podemos señalar algunos parámetros que definen la calidad del servicio tanto para el audio como para el video. Entre los cuales mencionamos el retardo, la perdida de paquetes, el Jitter promedio; entre otros. De esta forma se establecen unas métricas como son el tiempo de retardo al efectuar un cambio de canal, la disponibilidad que tiene el canal, fallos en el cambio de canal, entre otras. IPTV tiene características que lo diferencian de otros servicios de difusión, entre las cuales están consumir un mayor ancho de banda y de esta forma requerir una conexión de alta velocidad en el tramo de acceso. (Song et al., 2009; Ferro y Cesar, 2011; Viloria et al., 2008; Avellaneda et al., 2014).

En un sistema IPTV el tiempo que transcurre desde que el usuario oprime el botón para cambiar de canal, hasta que el canal solicitado es mostrado en el monitor es bastante extenso. Este retardo generado ha sido un gran inconveniente para los proveedores de TV a través de redes IP. Uno de los aspectos importantes de la entrega de contenido transmitido en vivo a través de una red IP es la velocidad a la que los usuarios finales pueden cambiar de canal durante la experiencia de ver televisión. La reducción del tiempo en el cambio de canal (más conocido por su término en Inglés Channel Zapping Delay o CZD), es un problema crítico que está afectando la calidad de experiencia del usuario con los servicios de IPTV (Manzato y Fonseca, 2013; Ramos, 2013; Cho et al., 2004; Cuellar et al., 2014).

Los proveedores de servicios de telecomunicaciones en Colombia que ofrecen aplicaciones de video como IPTV, han desarrollado estrategias tecnológicas que garantizan al usuario final una mejor calidad de servicio, aprovechando sustancialmente los recursos de la red; Sin embargo debido a diferentes factores como la complejidad en el comportamiento del video, la heterogeneidad de las diferentes tecnologías de acceso, entre otros. Han hecho que no se pueda garantizar una total calidad en cuanto a experiencia del usuario, como es el caso en el retardo de cambio de canal. De esta forma se pretende analizar el comportamiento del trafico IPTV sobre las diferentes tecnologías de acceso que ofrecen los ISP en Colombia, evaluando los parámetros de calidad de servicio que repercuten sobre el efecto "Channel Zapping Delay". (Martelo et al., 2015; Bonastre et al., 2011).

Las plataformas tecnológicas en que se basa la televisión convencional por cable, implementando los receptores satelitales, cuenta con la capacidad de recibir todos sus servicios televisivos de una forma simultánea, mientras que por otro lado los sistemas IPTV realizan la gestión del flujo de los canales individualmente, la selección de otro canal generará un nuevo flujo de información y por lo tanto un retardo en la transmisión de la señal. Esto desde la percepción de calidad de experiencia del usuario mostraría un retardo de tiempo en el cambio de canal (Manzato y Fonseca, 2013; Sánchez, 2008). Para mejorar la calidad de servicio en un sistema IPTV y en una transmisión de video en general, es fundamental conocer y evaluar su comportamiento a través de la red.

De acuerdo con lo anterior, el presente trabajo tiene por objetivo realizar el modelado del retardo del cambio de canal en una transmisión IPTV que permitan evaluar de una manera más precisa el comportamiento del video (Tanwir y Perros, 2013; Santos, 2009). El aporte principal de esta investigación radica inicialmente en poder determinar el comportamiento del retardo generado por la red en el momento de realizar una solicitud de cambio de canal, teniendo en cuenta cada una de las etapas donde el servicio pudiera estar disponible. Después de realizadas las mediciones de este retardo se quiso efectuar un aporte mostrando a través de dos modelos de series de tiempo, de qué manera se podía llegar a reducir el error promedio de dicho retardo, y a su vez determinar cuál de los dos modelos era más eficiente en cada una de las etapas planteadas.

Para desarrollar el modelo propuesto, inicialmente se diseñó un escenario de simulación de una red IPTV, considerando un esquema jerárquico desde el cliente hasta la cabecera. Para el desarrollo de la simulación se plantea un escenario con una topología de 20 usuarios. En la primera parte se tiene la red del cliente, conformada por 20 cajas receptoras (STB) y 10 dispositivos Gateway (HG), los cuales hacen de puente entre la red de acceso y la red doméstica. Por cada HG se conectan 2 dispositivos STB. En la red de acceso se encuentran los multiplexores de acceso a línea de abonado o dispositivos DSLAM, cada dispositivo recibe el flujo de 5 Gateway, y se dispone de dos DSLAM para la red planteada. Para comunicar la red de acceso con la red de distribución o el Core, se dispone de un enrutador de borde LHR, el cual recibe todo el flujo multicast de los dos dispositivos DSLAM. En la red de transmisión se cuenta con un enrutador de borde FHR, encargado de comunicar la red de distribución con la cabecera IPTV, este enrutador recibe todos los servicios de flujo multicast entregado por los dispositivos de la cabecera (HEADEND). 
La simulación del sistema IPTV planteado se realizó a partir del software de simulación de redes NS2 y EI tiempo de simulación establecido fue de 10 segundos. Cuando el cliente se dispone a realizar una solicitud de cambio de canal desde el control remoto, tiene dos opciones: solicitar un canal adyacente (+ -) o un canal aleatorio, oprimiendo simplemente el número de canal. Con base en esto se han planteado 4 escenarios de simulación, para los cuales se ha tenido en cuenta cada una de las etapas de transmisión desde el usuario hasta la cabecera, así como el tipo de congestión utilizado, para este requerimiento se tiene en cuenta dos estados: con o sin congestión en la red. Finalmente, para el modelado del retardo del cambio de canal en el sistema IPTV planteado, se efectuó el análisis y comparación de diferentes modelos correlacionados, basados en series de tiempo univariadas: modelo Autorregresivo (AR) y modelo de Promedios Móviles (MA).

En la patente (Lundqvist, 2011) se plantea un método en un servidor para proporcionar un rápido cambio de canal (FCC) para una red IPTV, en el que el FCC a otro canal, es facilitado a través de un flujo de unidifusión de dicho canal antes de unirse a un flujo de multidifusión, en donde la información de latencia de red es conocida y la determinación del retardo del FCC del flujo de multidifusión depende del valor del último retardo de la red. El servidor cuenta con un procesador configurado para determinar el retardo del FCC del flujo de multidifusión con respecto al flujo de multidifusión original. En este caso es importante conocer el retardo del FCC o modelar su comportamiento como se muestra en los resultados de este trabajo.

En (Ramos, 2009) los autores adoptan un distribución Gamma con k= 0.5 (parámetro de escala) y $\theta=5.8$ (parámetro de forma) para modelar el cambio de canal. Los resultados muestran que la mayoría de los cambios de canal durante el zapping son secuenciales. Dicho comportamiento puede ser usado para construir una arquitectura de red novedosa que pueda reducir el retardo en la experiencia de los usuarios de IPTV. Para ese propósito los autores proponen calcular la probabilidad de que el usuario de IPTV regrese al canal que estaba viendo antes de realizar el cambio de canal (Ramos, 2009).

En (Chae, 2010) los autores analizan el comportamiento del cambio de canal de los usuarios a través de un proceso de Semi-Markov, con el cual estiman el tiempo promedio de cambio de canal y el uso medio de ancho de banda, para determinar el número eficiente de canales precargados en un periodo de visualización y posteriormente seleccionar dichos canales teniendo en cuenta las preferencias del usuario. Los autores concluyen que el número de operaciones del cambio de canal, antes de la visualización del canal, sigue una distribución de Poisson con una media de 3.7. El resto del artículo está estructurado como sigue. En la sección II se realiza una descripción del retardo en el cambio de canal en una transmisión IPTV, analizando las causas que generan el retardo y las etapas del proceso. En la sección III se describe el diseño y la simulación de la red IPTV a través de cuatro escenarios que permitan realizar un análisis más detallado. En la sección IV se desarrollan los modelos de series de tiempo. En la sección V se presenta el análisis de resultados para cada escenario y modelo planteado. Finalmente en la sección VI se presentan las conclusiones.

\section{RETARDO DEL CAMBIO DE CANAL EN UNA TRANSMISIÓN IPTV}

En la televisión broadcast se puede ajustar el sintonizador a la señal en un solo cuadro de tiempo y reproducir el video seguidamente, por lo que los tiempos de Zapping son mínimos, por lo tanto, el cambio de canal no tiene impacto en la calidad de experiencia del usuario. Sin embargo, en el caso de IPTV el flujo de información llega individualmente para cada canal, por lo que al realizar un cambio de canal se debe cancelar el flujo enviado del canal anterior y enviar el nuevo flujo de datos del canal a desplegar. El proceso de cambio de canal se lleva a cabo en el servidor en lugar de los set-top box, que es el caso de los entornos tradicionales de transmisión de redes de televisión. Los usuarios finales esperan a ser capaz de cambiar rápidamente de canal en sus televisores. Debido al hecho de que el set-top box interactúa con la red durante un cambio de canal, las latencias de la experiencia de visualización son una posibilidad. Además de las demoras de los equipos en el hogar digital, la red y el centro de IPTV de datos puede introducir los posibles retrasos en el proceso de cambio de canal (Manzato y Fonseca, 2013; Eunji et al., 2014; Moumtadi et al., 2008).

En (Lloret, 2011) se realiza una contextualización de la media de los parámetros QoE, los cuales miden la experiencia del usuario respecto a los efectos generados por los parámetros QoS. El autor expresa que los parámetros QoE se pueden medir en la capa de transporte y la capa de aplicación de una red TCP/IP. Aunque hay muchos parámetros subjetivos que pueden ser incluidos en el QoE, como la disponibilidad de contenidos, la facilidad y disposición de la indexación de contenido, interfaz de usuario, la ergonomía, el diseño de navegación y guía de programación. Según el autor Zapping es una de las áreas principales donde la calidad de la experiencia del usuario en una transmisión IPTV se puede medir objetivamente, así como verificar que se está recibiendo la trama correcta. El Zapping muestra la rapidez con que los clientes cambian a un canal. Un retraso de 1s se considera como zapping aceptable, y entre 100 y $200 \mathrm{~ms}$ se considera instantánea 
Dentro de una transmisión IPTV existen diferentes sistemas que aportan un grado de retardo a la hora de realizar el cambio de canal. En Fig. 1 se puede apreciar cada una de las etapas que influyen en el CZD, desde que se realiza la solicitud por parte del usuario hasta que se visualiza el servicio en el TV.

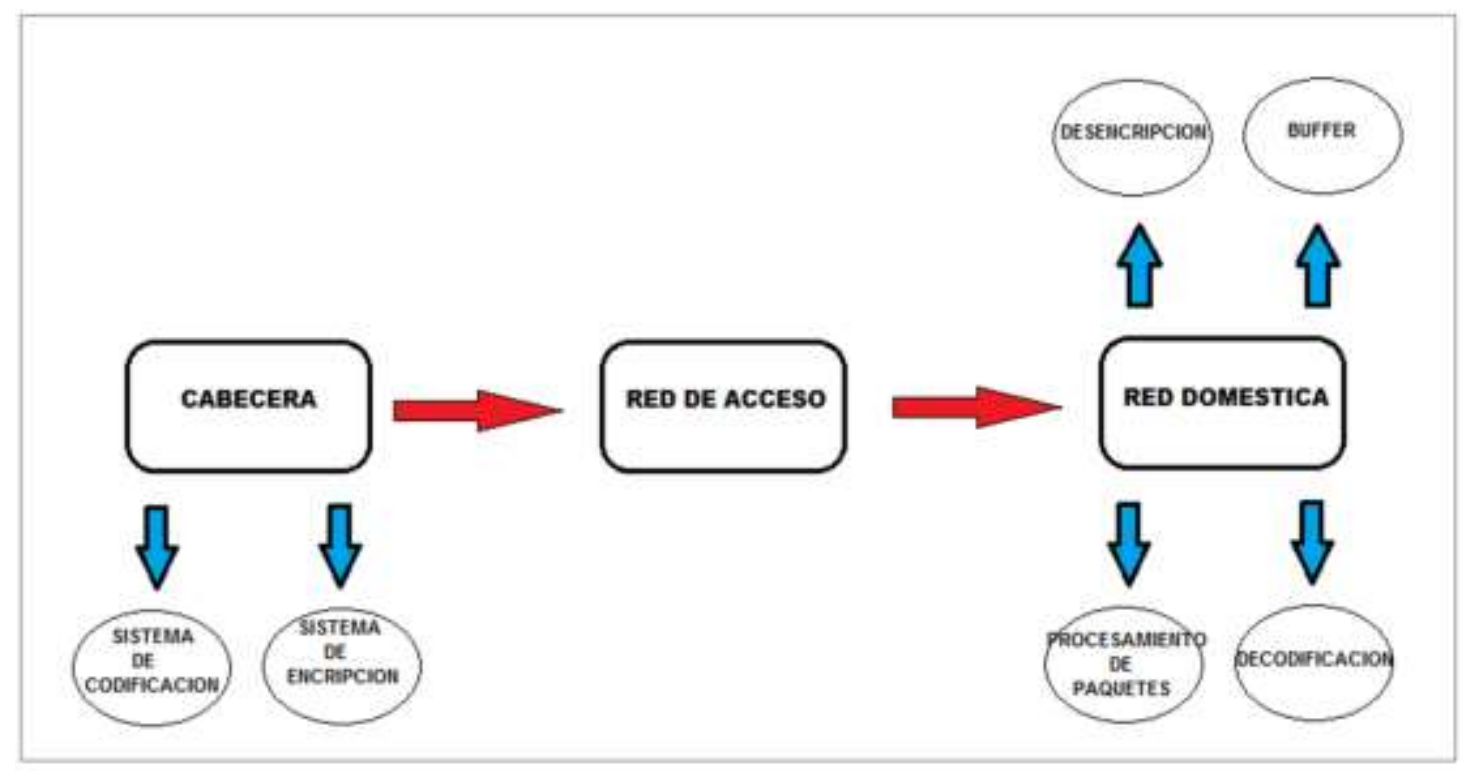

Fig. 1. Etapas que afectan el retardo en el cambio de canal

Cada una de las etapas mostradas en la Fig. 1 genera de una u otra forma un retardo a la hora de enviar la solicitud de cambio de canal. Por ejemplo, la decodificación de la señal comprimida en el STB juega un papel importante en el retraso que se experimenta en el proceso de cambio de canal. En el momento que se usa el algoritmo de compresión MPEG, el STB tiene que esperar a que una trama I en el stream de entrada tenga que esperar antes de comenzar la decodificación. La trama I define un grupo de imágenes (GOP) y contiene toda la información necesaria requerida para reconstruir la imagen codificada. Esto no tiene ninguna dependencia de las tramas anteriores, lo cual reduce el efecto de las tramas perdidas. El periodo de espera para la trama I dependerá del número de tramas I transmitidas por segundo (Moumtadi et al., 2008; Video Services Forum, 2006). Para nuestro caso donde solo se va tener en cuenta el retardo generado en el proceso de transmisión, se va trabajar con los protocolos Multicast IGMP y PIM.

\section{Pasos para el proceso de cambio de canal en un sistema IPTV}

En la primera etapa se exponen los componentes de recepción, donde la puerta de enlace del Home Gateway (HG) contiene el flujo de todos los Sep Top Box (STB) conectados a él. Siguiendo una topología jerárquica, el multiplexor de acceso a la línea de abonado digital (DSLAM), a su vez recibe el flujo de todos los HG conectados directamente a él. Por último está el Last Hop Router (LHR) quien concentra el flujo de los DSLAM involucrados. Independiente de la arquitectura del core, en la etapa de transmisión el First Hop Router (FHR) recibe el flujo de todos los canales del sistema IPTV, mientras la cabecera (IPTV HEADEND) se encarga de administrar, gestionar y distribuir los diferentes contenidos del sistema IPTV. En la Fig. 2 se muestra cada uno de los componentes en una arquitectura IPTV (Joskowicz y Sotelo, 2005).

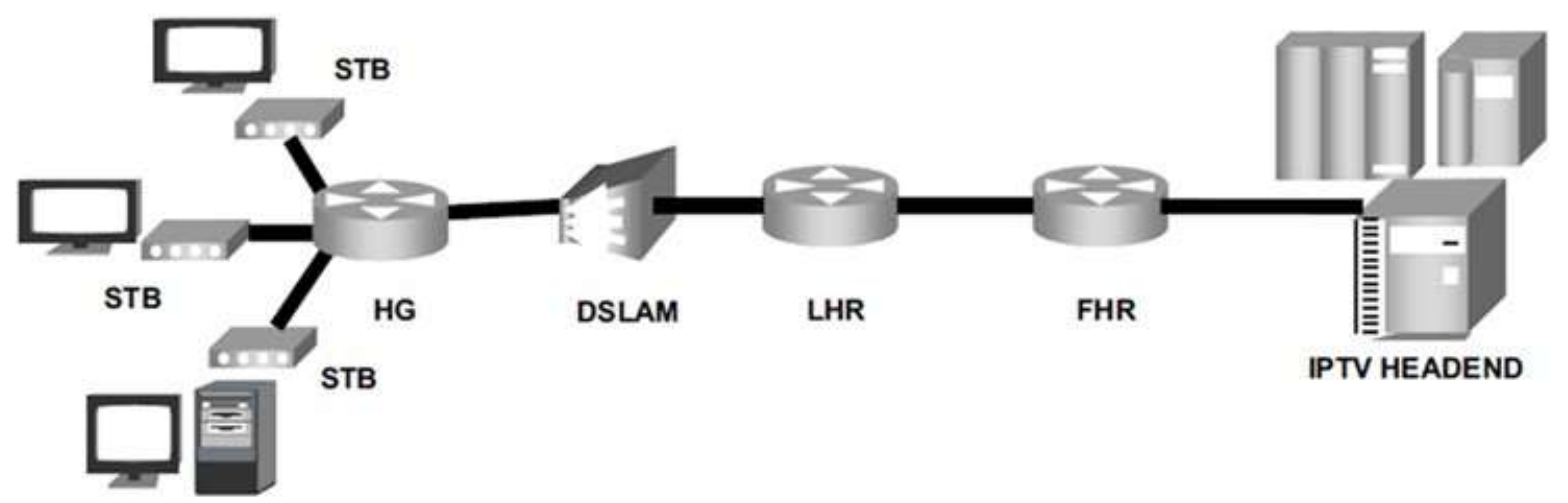

Fig. 2: Arquitectura de un sistema IPTV. (Joskowicz y Sotelo, 2005). 
El proceso para el cambio de canal en una transmisión IPTV se puede apreciar en la Fig. 3. Para la unión a un flujo multicast el STB utiliza el protocolo de gestión de grupos en internet (IGMP), que se encarga de enviar mensajes de entrada y abandono del grupo. De esta forma al usuario realizar una solicitud de cambio de canal hacia el STB utilizando el control remoto, este requerimiento es procesado por el STB y si el canal se encuentra disponible en el dispositivo, este comienza a decodificarlo y a mostrar el video en el TV. Si no está disponible el STB envía a la red un mensaje IGMP Leave para el canal que se encuentra actualmente y un mensaje IGMP Join para el canal solicitado. De esta forma el HG recibe la solicitud IGMP actuando como si fuera el mismo un host, si el canal solicitado se encuentra en el Home Gateway este copiara el flujo multicast y lo retransmitirá al STB inmediatamente. Si no está disponible el proceso anterior se repite para el DSLAM y el LHR. EI LHR igual que los dispositivos anteriores debe utilizar IGMP para comunicarse con el DSLAM, pero a su vez debe manejar el protocolo multicast independiente de baja densidad (PIM-SM) para comunicarse con el FHR a través del core de la red. El LHR también envía mensajes PIM-SM al FHR para notificarle que el estado de membrecía a grupos multicast ha cambiado, consiguiendo así mismo que el flujo multicast correspondiente a un servicio se detenga o se transmita un flujo nuevo. Luego que el flujo IPTV atraviesa toda la red, este es recibido por el STB, el cual debe esperar al siguiente frame I para empezar a llenar el buffer y luego decodificar la señal (Cho et al., 2004; Díaz y Merino, 2010).

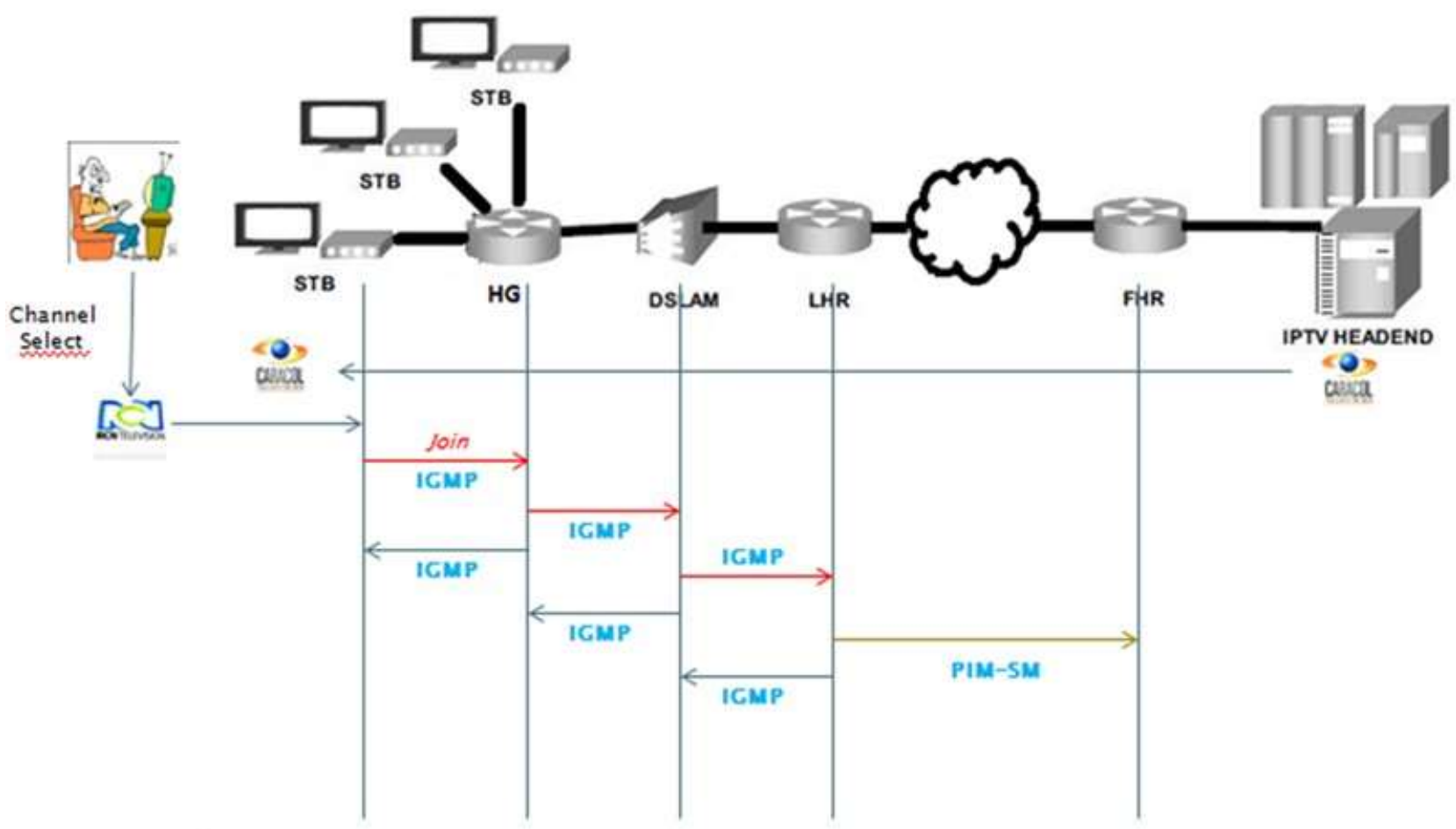

Fig. 3. Pasos para el proceso de cambio de canal en un sistema IPTV.

\section{DISEÑO Y SIMULACION DE LA RED IPTV}

En la Fig. 4 se puede observar la arquitectura planteada para la red IPTV, considerando un esquema jerárquico desde el cliente hasta la cabecera. Para el desarrollo de la simulación se plantea un escenario con una topología de 20 usuarios. En la primera parte se tiene la red del cliente, conformada por 20 cajas receptoras (STB) y 10 dispositivos Gateway (HG), los cuales hacen de puente entre la red de acceso y la red doméstica. Por cada HG se conectan 2 dispositivos STB. En la red de acceso se encuentran los multiplexores de acceso a línea de abonado o dispositivos DSLAM, cada dispositivo recibe el flujo de 5 Gateway, y se dispone de dos DSLAM para la red planteada. Para comunicar la red de acceso con la red de distribución o el Core, se dispone de un enrutador de borde LHR, el cual recibe todo el flujo multicast de los dos dispositivos DSLAM. En la red de transmisión se cuenta con un enrutador de borde FHR, encargado de comunicar la red de distribución con la cabecera IPTV, este enrutador recibe todos los servicios de flujo multicast entregado por los dispositivos de la cabecera (HEADEND). 


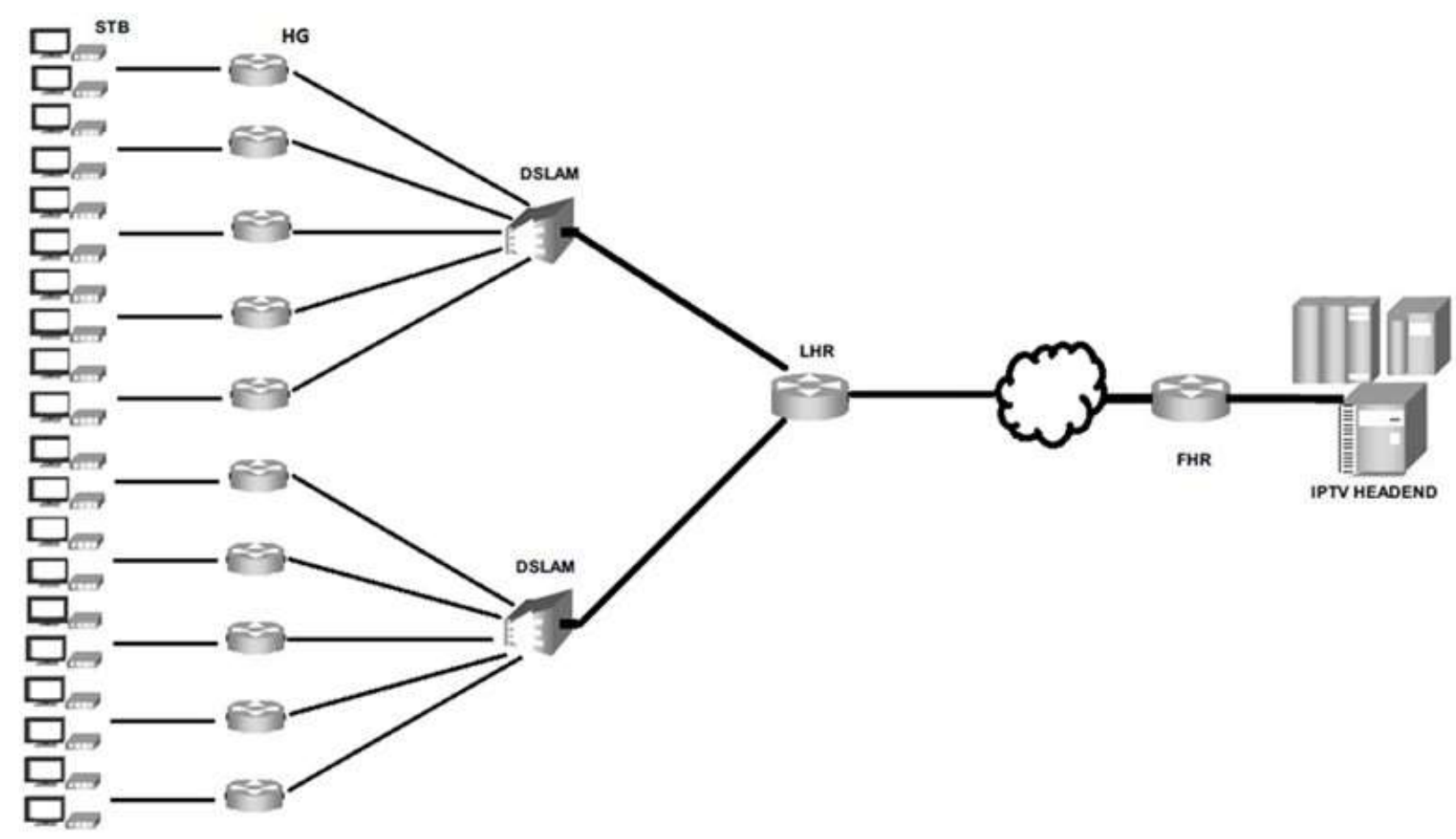

Fig. 4: Esquema de la arquitectura IPTV planteada.

Para el desarrollo de la simulación se configuraron 44 nodos, los cuales están distribuidos de la siguiente manera: Nodos 0-19: Dispositivos Set Top Box (STB); Nodos 20-29: Dispositivos Home Gateway (HG); Nodo 30-31: Dispositivos DSLAM; Nodo 32: Ultimo enrutador de borde (LHR); Nodo 33-40: Enrutadores Core MPLS; Nodo 41: Primer enrutador de borde (FHR); Y Nodo 42-43: Servidores IPTV.

Después de descritos cada uno de los nodos se establecen los enlaces dúplex con un ancho de banda de $12 \mathrm{Mbps}$ para la red doméstica implementado tecnología ADSL2 y con un droptail de $10 \mathrm{~ms}$. A su vez se implementa un enlace de fibra entre la red de acceso y el LHR con el mismo tiempo de encolamiento. El Core MPLS se configura con un ancho de banda de $1 \mathrm{~Gb}$ y un droptail de $1 \mathrm{~ms}$, utilizando enrutadores LSP Y LER. De igual forma entre el core y el enrutador de borde de la cabecera se implementa un enlace de fibra full dúplex de 1 Gbps. Posteriormente se definen la configuración de las fuentes de tráfico establecidas mediante tráfico CBR y paquetes nulos para la asignación de espacios de memoria.

\section{Escenarios de simulación}

A partir del esquema de la arquitectura IPTV diseñada, se han planteado 4 escenarios de simulación, para los cuales se ha tenido en cuenta cada una de las etapas de transmisión desde el usuario hasta la cabecera, así como el tipo de congestión: con o sin congestión en la red.

Primer Escenario: Para el análisis de este escenario se tendrán en cuenta los estudios efectuados de grupos de canales adyacentes, por lo tanto la medición del retardo será efectuada dentro de la red del hogar. De esta forma la solicitud se efectuará de un STB al otro STB teniendo como puente de comunicación el HG.

Segundo Escenario: Si al efectuar la solicitud de cambio de canal, este servicio no se encuentra almacenado en el buffer de un STB de la misma red doméstica, la solicitud se realizará hasta otro dispositivo STB ubicado geográficamente en la misma zona e interconectado por el mismo DSLAM. La transmisión se efectúa pasando por el Home Gateway hacia el dispositivo DSLAM, encargado de distribuir la red de acceso o última milla. Y nuevamente del DSLAM al HG del dispositivo STB que contiene el servicio. EI DSLAM se encarga de distribuir los diferentes grupos multicast en una zona geográficamente definida.

Tercer Escenario: En esta fase se mide la trasmisión desde el dispositivo STB hasta el LHR, el cual es el enrutador de borde del Core en la parte de la red de acceso. Atravesando por cada uno de los dispositivos de la cadena. Este enrutador se encarga de almacenar los Join leave de toda la red de acceso. 
Cuarto Escenario: En la cuarta etapa se define la transmisión desde el STB del usuario que efectuó la solicitud hasta el enrutador de borde de la cabecera (FHR). Pasando por el Core, donde se implementa tecnología MPLS y utilizando el protocolo PIM - SM.

En el análisis de resultados se mostrará el retardo medido en cada escenario con y sin congestión en la red para cada uno de los cuatro escenarios.

\section{MODELOS DE SERIES DE TIEMPO}

Las series de tiempo tienen como objetivo central desarrollar modelos estadísticos que expliquen el comportamiento de una variable aleatoria que varía con el tiempo permitiendo estimar pronósticos futuros de dicha variable aleatoria (Álzate, 2004; Pagan et al., 1995).

Debido a que las series de tiempo son modelos idóneos para series correlacionadas y dado que varias investigaciones han demostrado que el tráfico de video es correlacionado, se decidió seleccionar los modelos AR y MA.

\section{Modelo Autorregresivo (AR)}

Un modelo AR describe una clase particular de proceso en que las observaciones actuales, $t$, son predecibles, en mayor o menor grado, de las observaciones u outputs previos. Por tanto, mediante el modelo Auto-regresivo se describe un proceso en que las observaciones actuales se hallan determinadas 0 influidas por las observaciones anteriores. Los modelos autorregresivos se basan en la idea de que el valor actual de la serie $Z_{t}$, puede explicarse en función de valores pasados $Z_{t-1}, Z_{t-2} \ldots . Z_{t-p}$, donde $p$ determina el número de rezagos necesarios para pronosticar un valor actual. El modelo autorregresivo de orden $p$ está dado por la ecuación (1):

$Z_{t}=C+\varphi_{1} Z_{t-1}+\varphi_{2} Z_{t-2}+\cdots+\varphi_{p} Z_{t-p}+a_{t}$

Donde $\varphi_{1} \ldots \varphi_{p}$ son los parámetros del modelo, $C$ es una constante y $a_{t}$ es un término de error o proceso de ruido blanco gaussiano. El proceso Autorregresivo es un modelo de regresión en el que las variables explicativas son la misma variable dependiente retardada. Una condición para que un modelo Autorregresivo sea un proceso estacionario es cuando $\varphi_{p}<1$. (Garrett y Willinger, 1994).

\section{Modelo de promedios móviles (MA)}

El modelo de promedios móviles supone que todas las observaciones de la serie de tiempo son igualmente importantes para la estimación del parámetro a pronosticar (en este caso el retardo). De esta manera, se utiliza como pronóstico para el siguiente periodo, el promedio de los $n$ valores, de los datos más recientes de la serie de tiempo. El modelo MA es determinado por una fuente externa. Estos modelos suponen linealidad, el valor actual de la serie, $X_{t}$ está influenciado por los valores de la fuente externa (Garrett y Willinger, 1994). El modelo de promedio móviles de orden q está dado por la ecuación (2).

$X_{t}=\theta_{0}-\theta_{1} \varepsilon_{t-1}-\theta_{2} \varepsilon_{t-2}-\cdots-\theta_{q} \varepsilon_{t-q}-\varepsilon_{t}$

Donde $\varepsilon_{t}$ es un proceso de ruido blanco y $\theta_{1} \ldots . \theta_{q}$ son los parámetros del modelo.

\section{ANÁLISIS DE RESULTADOS}

Para la evaluación del retardo inicialmente se han tomado 1000 muestras de cada variable en $\mathrm{t}$ instantes de tiempo, generadas a través del software NS2. Las figuras y mediciones del retardo en cada escenario se han desarrollado en MATLAB.

La Fig. 5 muestra el retardo medido en cada uno de los cuatro escenarios, sin congestión en la red. En el primer escenario como se menciona anteriormente se hace el análisis del retardo en la red doméstica teniendo en cuenta que el servicio solicitado se encuentra cargado ya en el STB, o en su defecto en otro STB del mismo hogar, por lo tanto el retardo medido es de aproximadamente $40 \mathrm{~ms}$. Para el segundo escenario la configuración de simulación está dada dentro de una red gestionada por el mismo DSLAM, teniendo en cuenta que el servicio solicitado por parte del usuario se encuentra disponible en un STB ubicado en esta misma zona geográfica; de esta forma el retardo en este escenario es de aproximadamente $58 \mathrm{~ms}$. Siguiendo la secuencia de transmisión para una solicitud de cambio de canal para el tercer escenario 
la configuración está dada hasta el último enrutador de borde para el acceso al core (LHR); teniendo en cuenta que la velocidad de transmisión entre el DSLAM y el LHR es alta, la medición del retardo en este punto es similar al escenario 2 con un retardo de $56 \mathrm{~ms}$ aproximadamente. Finalmente se muestra el cuarto escenario, donde la solicitud de cambio de canal viaja hasta el enrutador de borde de la cabecera (FHR), en este escenario el retardo medido es de aproximadamente $90 \mathrm{~ms}$.

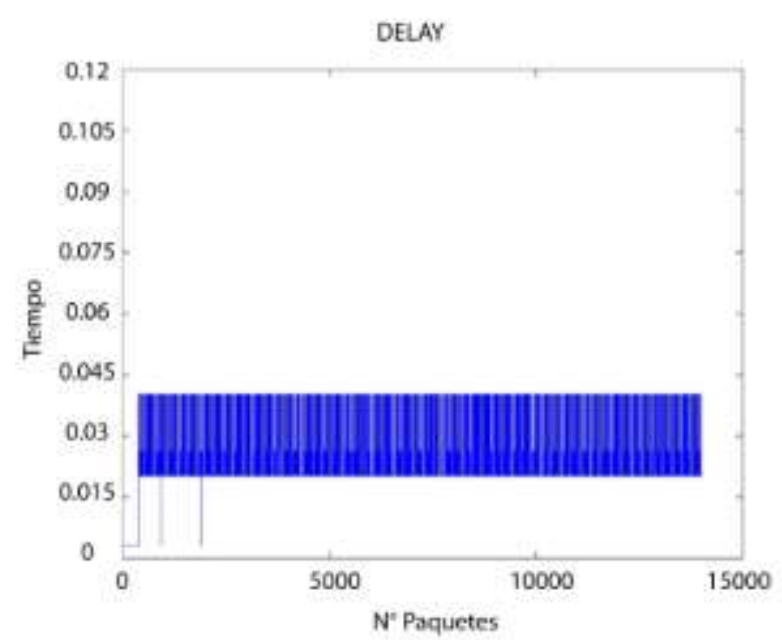

Escenario 1 sin congestión

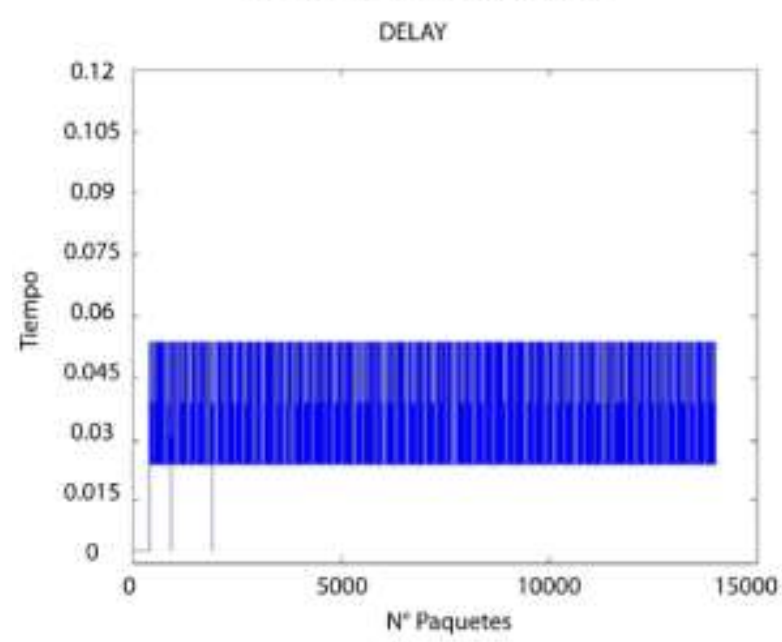

Escenario 3 sin congestión

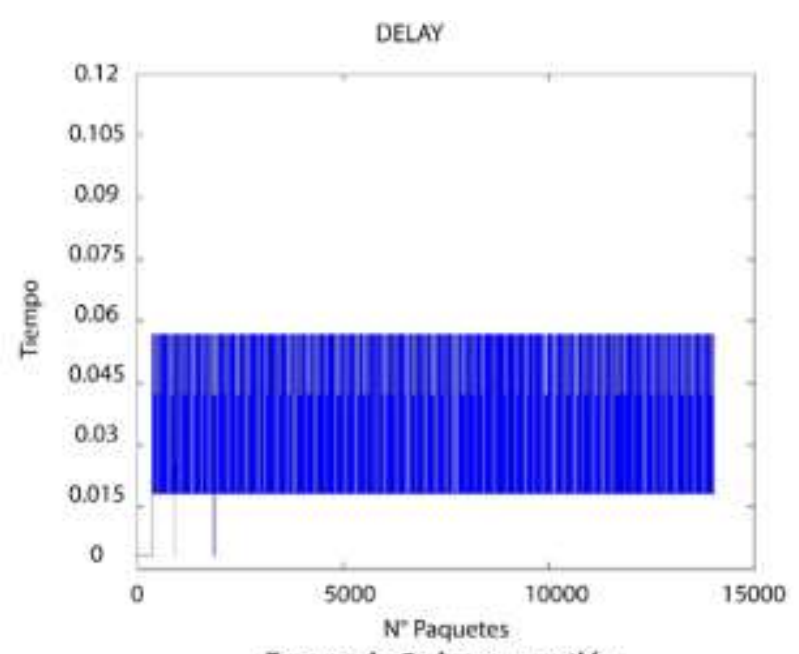

Escenario 2 sin congestión

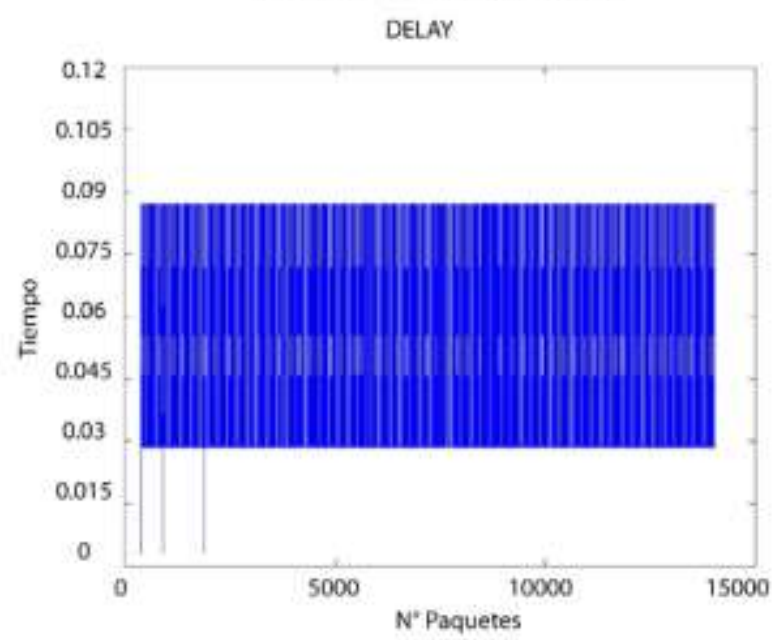

Escenario 4 sin congestión

Fig. 5. Retardo sin congestión en la red para los 4 escenarios

La Fig. 6 plantea los mismos cuatro escenarios de simulación pero con un valor agregado de congestión en la red. Las configuraciones de cada escenario son iguales a las planteadas en la Fig. 5; De esta forma el retardo aproximado se da de la siguiente manera: Escenario $1.40 \mathrm{~ms}$, Escenario $2.68 \mathrm{~ms}$, Escenario 3.63 ms y Escenario 4.104 ms.

En la segunda parte del análisis se realiza el modelado del retardo medido, con base en las dos series de tiempo seleccionadas. La base de datos para los 4 escenarios se ha desarrollado a través del software STATA.

\section{Modelo Autorregresivo (AR)}

Inicialmente se indica cual va ser la variable de tiempo en este caso un step de 1 a 1000 . Para la evaluación del modelo AR, como primera medida se realiza la autocorrelación parcial para el tráfico de paquetes por segundo (trafpaq). Esta autocorrelación parcial nos indica el orden (p) para un modelo AR. Con base en esto se realiza la estimación del trafpaq, y se estima el error absoluto y el error relativo. A continuación se muestra todo el proceso para el primer escenario.

A partir de los resultados obtenidos por la función de autocorrelacion parcial, se determina que el modelo autorregresivo es de orden 18, cuyo modelo inicial está dado por la ecuación (3). 


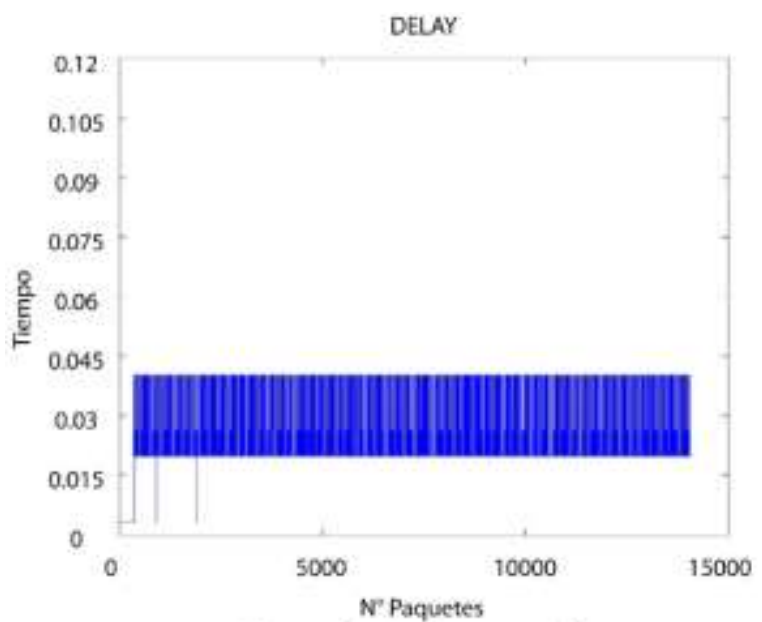

Escenario 1 con congestión

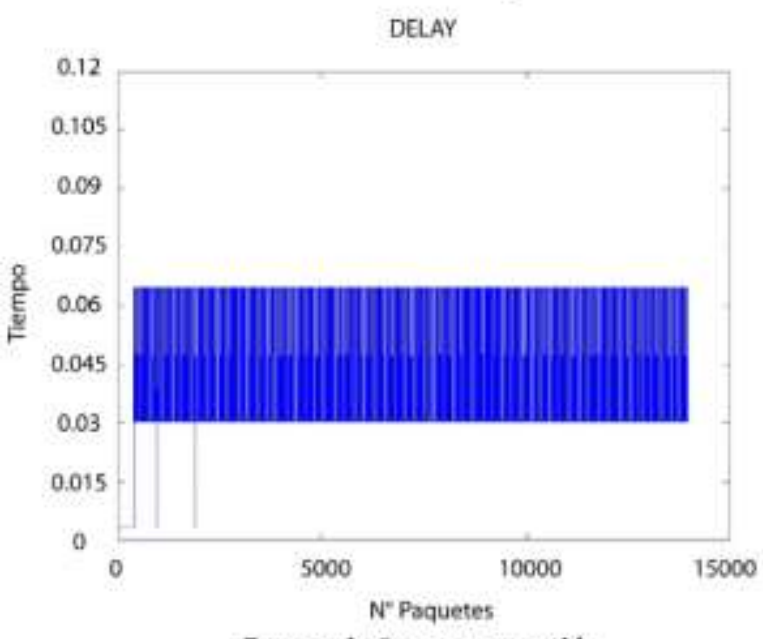

Escenario 3 con congestión

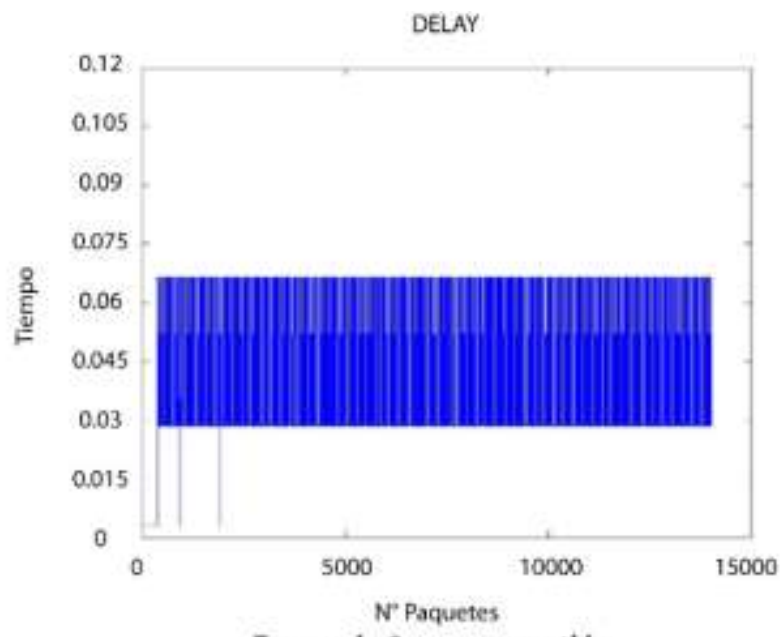

Escenario 2 con congestión

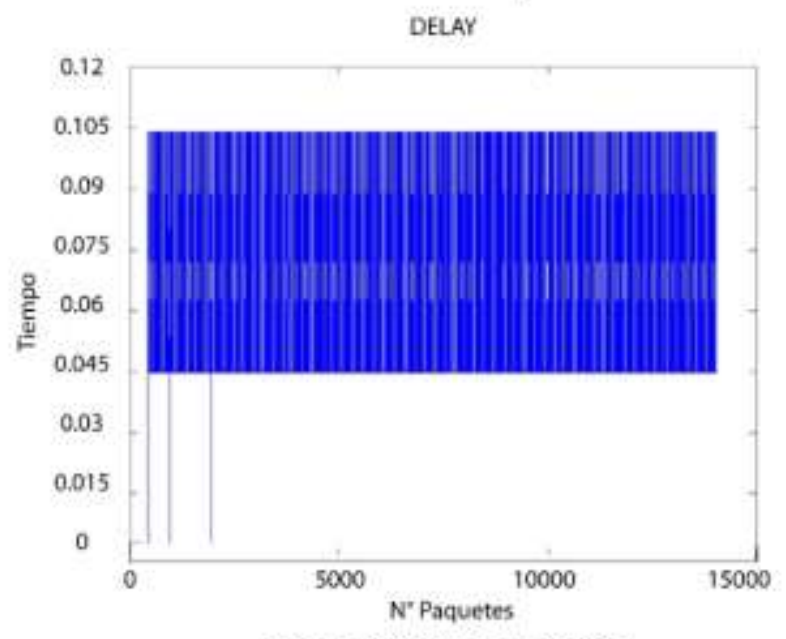

Escenario 4 con congestión

Fig. 6. Retardo con congestión en la red para los 4 escenarios

$Z_{t}=0,0211-0,1010{ }_{1} Z_{t-1}+0,1676_{2} Z_{t-2}+0,0654_{3} Z_{t-3}-0,1174_{4} Z_{t-4}+0,1258_{18} Z_{t-18}+a_{t}$

Después de calculados los valores estadísticos, se grafica la predicción del retardo para cada escenario. La Fig. 7 muestra la predicción para los 4 escenarios sin congestión en la red; en cada escenario se muestran 2 graficas: los datos de retardo medidos (color azul), comparados con los datos de retardo predichos (color rojo), donde se puede apreciar que la predicción del retardo que arroja la gráfica muestra los valores medios del retardo medido. De igual forma en la Fig. 8 se puede apreciar la predicción del retardo comparado con el retardo medido, en cada escenario, pero en este caso con la red congestionada.

\section{Modelo de promedios móviles (MA)}

Igualmente se configura un step de 1 a 1000. El algoritmo para este modelo es similar al modelo autorregresivo. Así mismo se realiza la estimación del trafpaq, y se estima el error absoluto y el error relativo. A partir de los resultados obtenidos por la función de autocorrelacion, se determina que el modelo de promedios móviles también es de orden 18, cuyo modelo inicial está dado por la ecuación (4).

$X_{t}=\left(1+0,114276_{1} L-0,181140_{2} L^{2}+0,081362{ }_{4} L^{4}+0,087489_{6} L^{6} 0,137221_{18} L^{18}\right) \times a_{t}$

De igual forma que en el modelo AR se calculan los valores estadísticos y se grafica la predicción del retardo en cada escenario. De la misma forma que en AR, solo se mostrará la ecuación del primer escenario. 


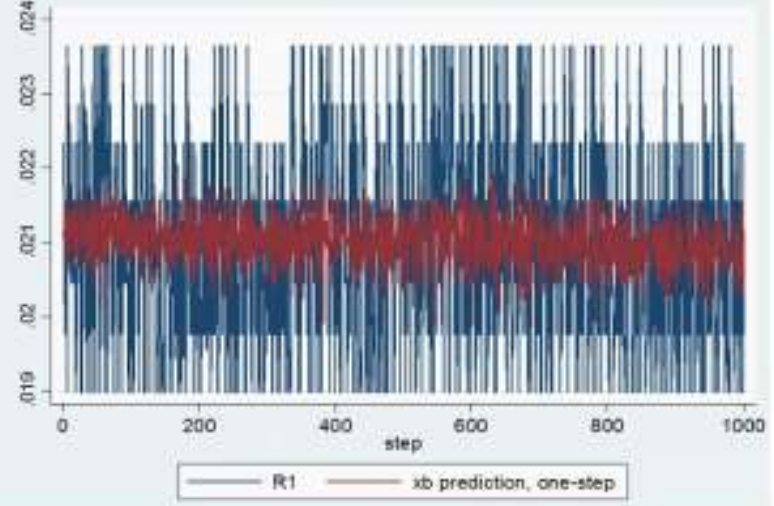

Predicción del retardo escenario 1

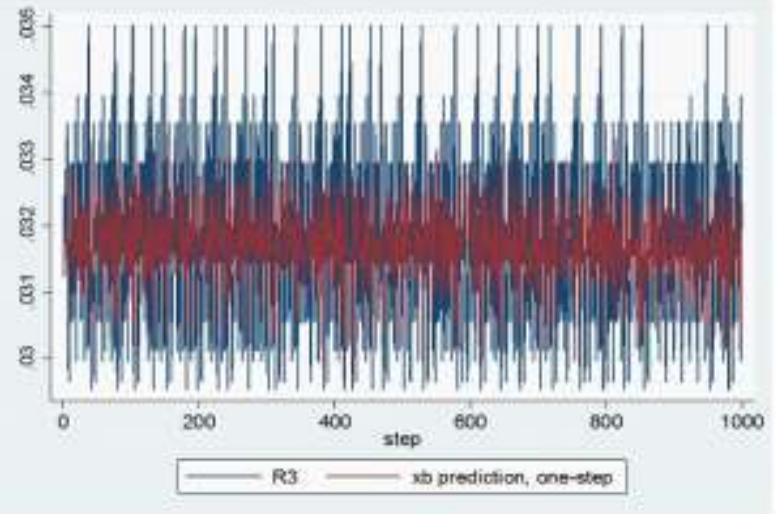

Predicción del retardo escenario 3

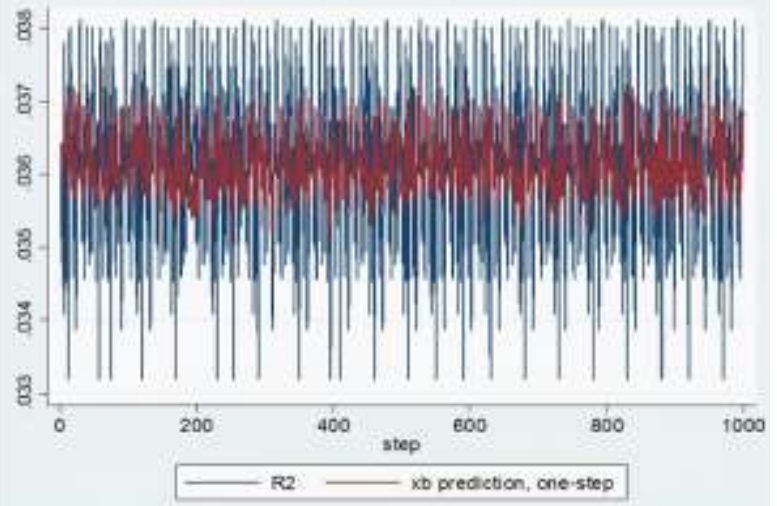

Precicción del retardo escenario 2

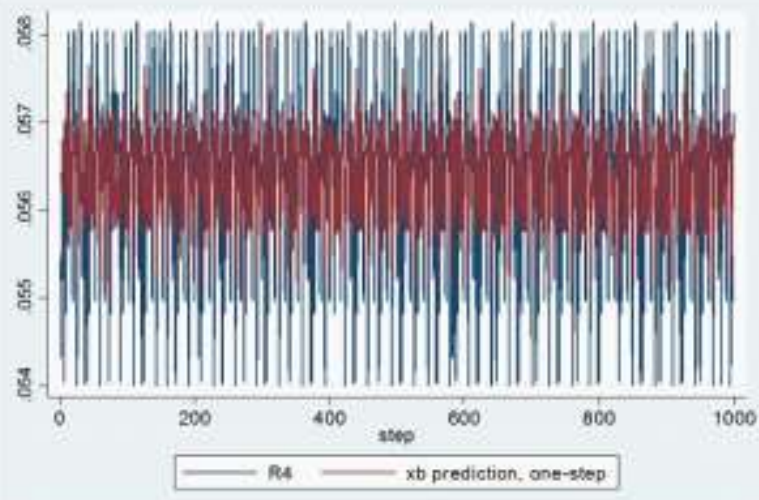

Predicción del retardo escenario 4

Fig. 7: Predicción del modelo AR para el retardo sin congestión de los 4 escenarios

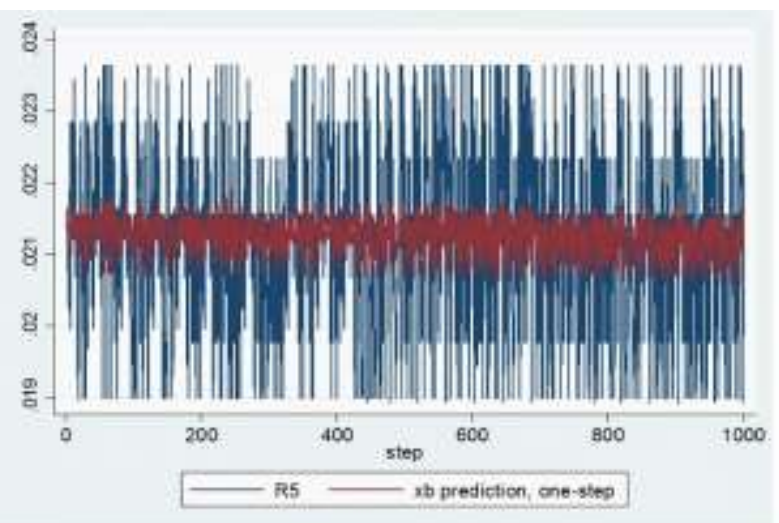

Predicción del retardo escenario 1

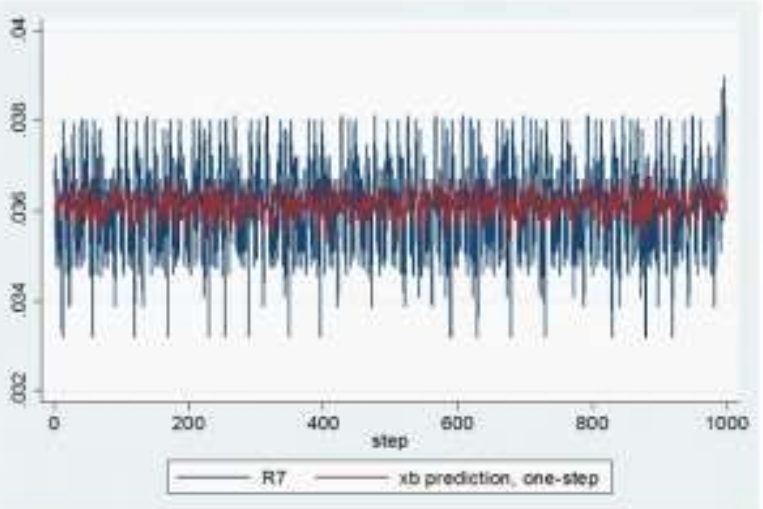

Predicción del retardo escenario 3

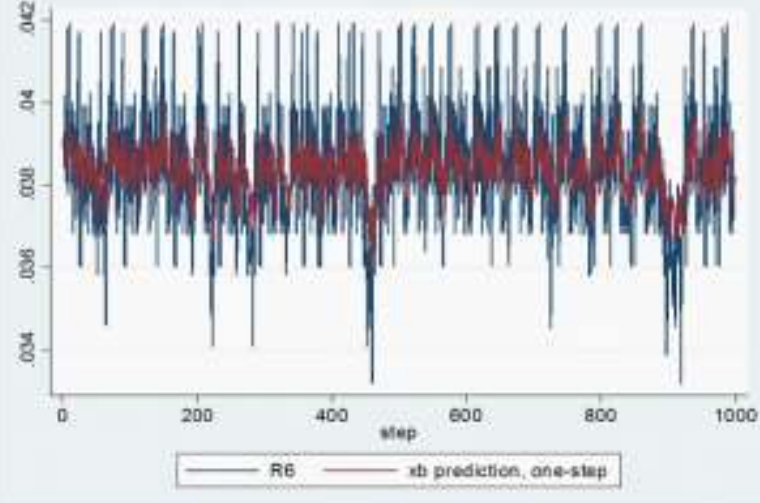

Predicción del retardo escenario 2

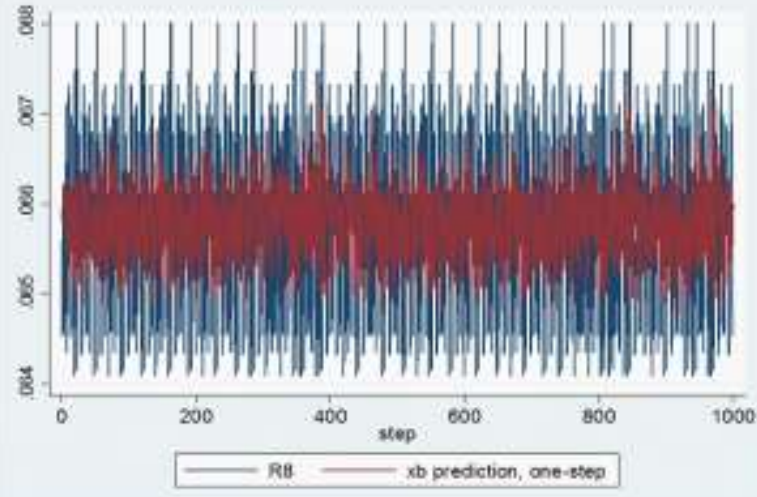

Predicción del retardo escenario 4

Fig. 8. Predicción del modelo AR para el retardo con congestión de los 4 escenarios 


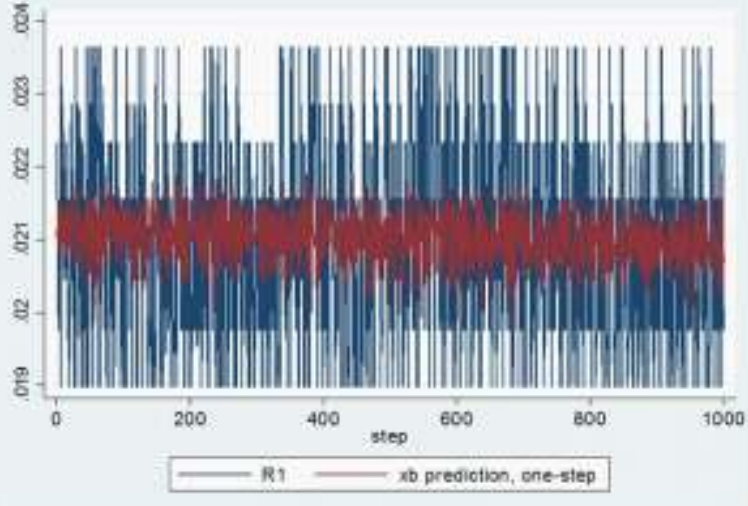

Predicción del retardo escenario

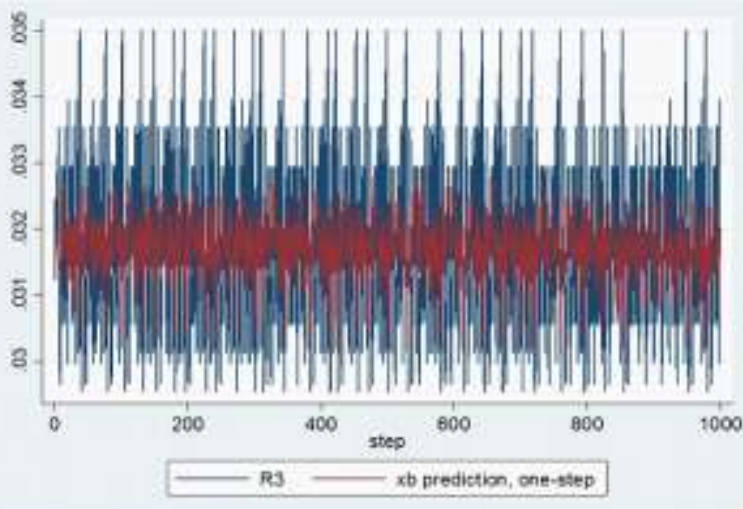

Predicción del retardo escenario 3

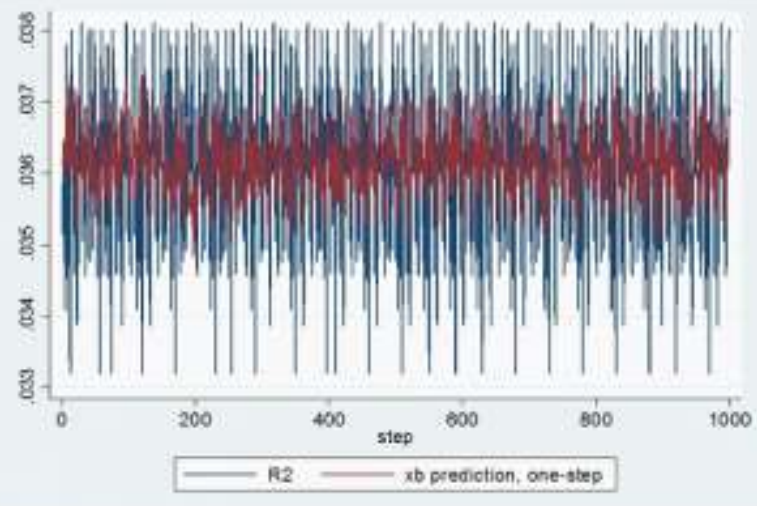

Predicción del retardo escenario 2

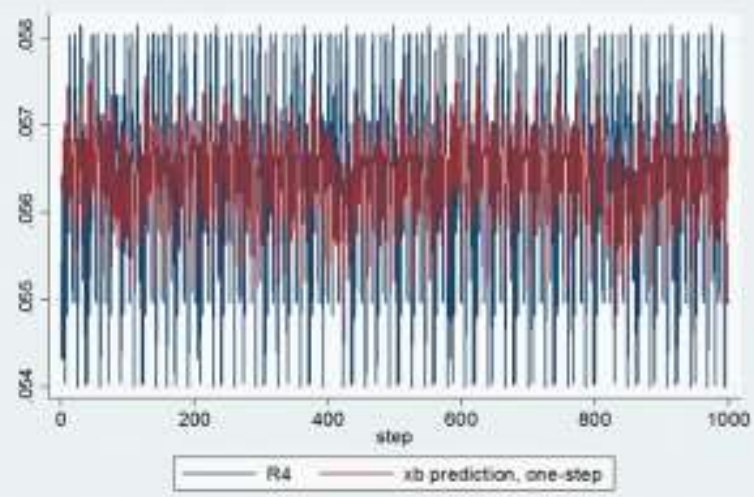

Predicción del retardo escenario 4

Fig. 9: Predicción del modelo MA para el retardo sin congestión de los 4 escenarios

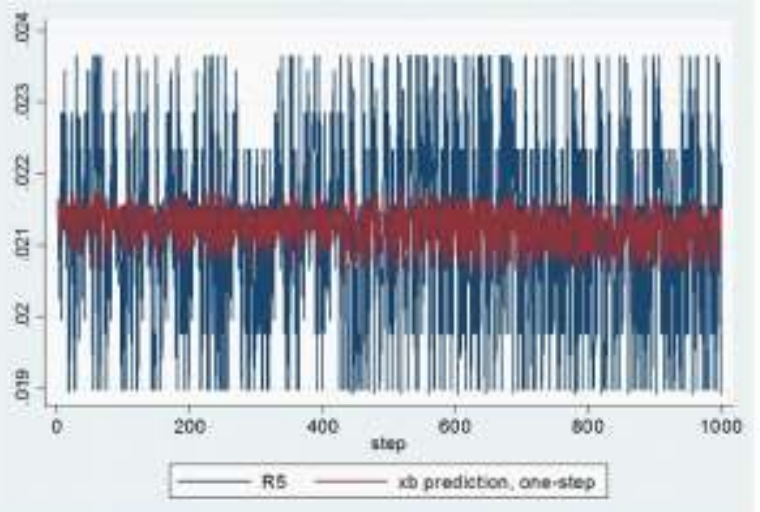

Predicción del retardo escenario 1

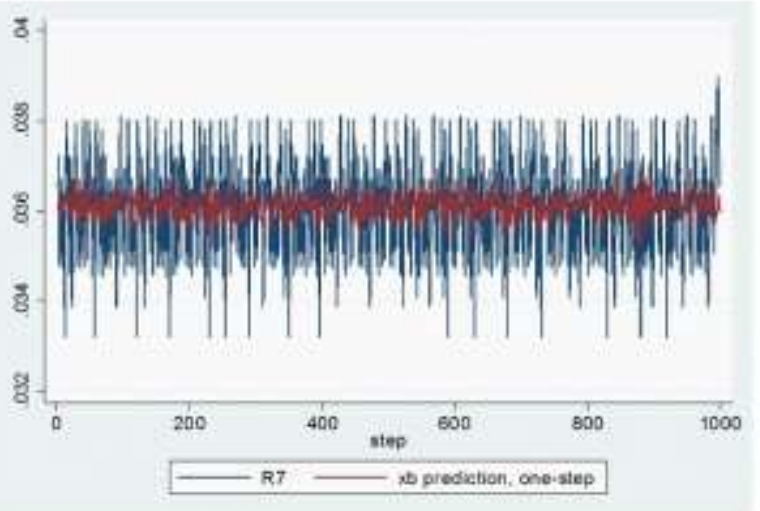

Predicción del retardo escenario 3

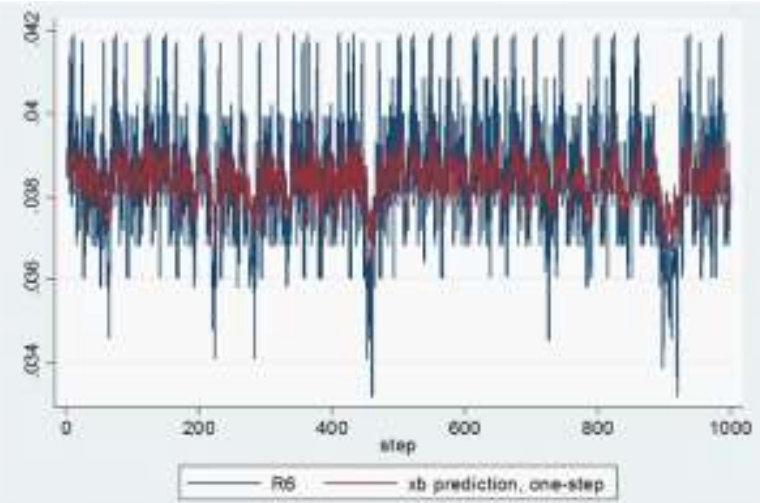

Precicción del retardo escenario 2

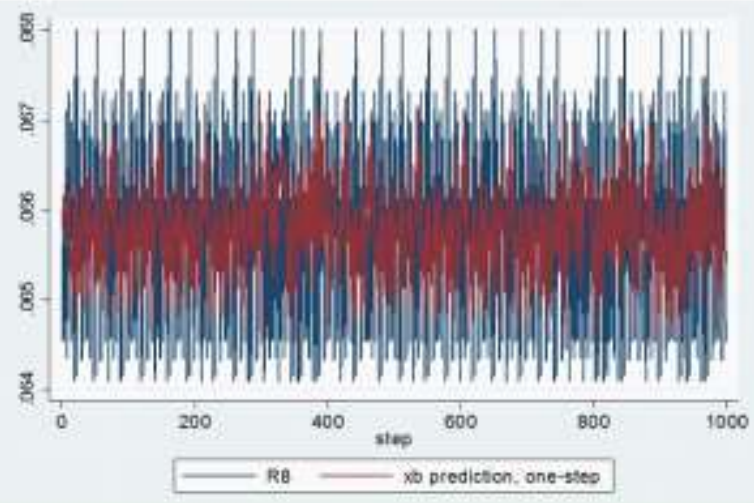

Predicción del retardo escenario 4

Fig. 10: Predicción del modelo MA para el retardo con congestión de los 4 escenarios 
La Fig. 9 describe la predicción en los 4 escenarios, sin congestión en la red, mientras la Fig. 10 describe la predicción en los 4 escenarios, con congestión en la red. La Tabla 1 describe la Relación del error promedio para los dos modelos de series de tiempo en cada uno de los escenarios planteados, tanto sin congestión como con congestión. Con base en el análisis realizado se puede apreciar que la diferencia entre cada dato no es muy significativa, sin embargo en la Tabla 2 se indicará el modelo que mejor se ajusta para cada escenario.

Tabla 1: Error promedio de los modelos AR y MA

\begin{tabular}{|c|c|c|}
\hline \multirow{2}{*}{ Escenarios } & \multicolumn{2}{|c|}{ Modelos de traficos } \\
\cline { 2 - 3 } & $\mathrm{AR}$ & $\mathrm{MA}$ \\
\hline 1 sin congestión & $6,01 \%$ & $5,99 \%$ \\
\hline 1 con congestión & $5,78 \%$ & $5,76 \%$ \\
\hline 2 sin congestión & $2,42 \%$ & $2,37 \%$ \\
\hline 2 con congestión & $2,95 \%$ & $2,97 \%$ \\
\hline 3 sin congestión & $3,28 \%$ & $3,34 \%$ \\
\hline 3 con congestión & $2,40 \%$ & $2,40 \%$ \\
\hline 4 sin congestión & $1,38 \%$ & $1,35 \%$ \\
\hline 4 con congestión & $1,16 \%$ & $1,20 \%$ \\
\hline
\end{tabular}

Tabla 2: Modelo que mejor se ajusta a cada escenario

\begin{tabular}{|c|c|}
\hline Escenario & Modelo \\
\hline 1 sin congestión & $\mathrm{MA}$ \\
\hline 1 con congestión & $\mathrm{MA}$ \\
\hline 2 sin congestión & $\mathrm{MA}$ \\
\hline 2 con congestión & $\mathrm{AR}$ \\
\hline 3 sin congestión & $\mathrm{AR}$ \\
\hline 3 con congestión & $\mathrm{MA}-\mathrm{AR}$ \\
\hline 4 sin congestión & $\mathrm{MA}$ \\
\hline 4 con congestión & $\mathrm{AR}$ \\
\hline
\end{tabular}

\section{CONCLUSIONES}

Existen 4 etapas en una transmisión IPTV donde un servicio puede permanecer en el momento en que un usuario hace la solicitud de cambio de canal. Cuando el servicio se encuentra en la red doméstica; segundo, en la red de acceso controlada por el mismo DSLAM; tercero, en el enrutador de borde de la red de acceso; y por último, en el enrutador de borde de la cabecera. Se realizó la evaluación de dos modelos de tráfico univariados basados en series de tiempo (modelo AR y modelo MA), con el fin de analizar el grado de autocorrelación de cada uno, en los diferentes escenarios planteados. Cuando la red se encuentra sin congestión, en promedio el modelo que más se ajusta es el de promedio móviles MA, mientras que cuando la red se encuentra congestionada, en promedio el modelo que más se ajusta es el modelo autorregresivo AR.

Si en el momento de efectuar la solicitud de cambio de canal, el servicio se encuentra en un dispositivo STB de la misma red del hogar, e independientemente si la red esta congestionada o no; el modelo que mejor se ajusta para modelar el retardo es el modelo MA.

Si en el momento de efectuar la solicitud, el servicio se encuentra disponible dentro de la misma red de acceso (DSLAM), y la red no está congestionada, el modelo que mejor se ajusta para modelar el retardo es el modelo MA.

Si en el momento de efectuar la solicitud, el servicio se encuentra disponible dentro de la misma red de acceso (DSLAM), y la red se encuentra con algún tipo de congestión, el modelo que mejor se ajusta es el autorregresivo.

Cuando el usuario realiza una solicitud de cambio de canal y el servicio está disponible en el enrutador de borde LHR y a su vez la red se encuentra sin congestión, el modelo que mejor ajusta el retardo en este escenario es el modelo autorregresivo. Pero si la red se encuentra con congestión, ambos modelos sirven. 


\section{REFERENCIAS}

Álzate, M. A.; Modelos de tráfico en análisis y control de redes de comunicaciones. Revista de ingeniería, 9(1), 63-87 (2004)

Arévalo, S.; Proyecto de tesis sobre sistemas IPTV. Quito: Escuela Politécnica Nacional, mayo (2010)

Avellaneda, Jaime V, Rodríguez, Jordi R. y López, Danilo A.; Servicios de Televisión sobre la Plataforma de Internet (IPTV-IMS) usando Protocolo de Flujo en Tiempo Real (RTSP) y Protocolo de Transferencia de Hipertexto (HTTP), doi: 10.4067/S0718-07642014000100008, Inf. Tecnol. (en línea), 25(1), 67-76 (2014)

Bonastre O. M., M. J. Montpetit y P. Cesar; Advances in IPTV technologies, Signal Processing: Image Communication, 26(7), 325-326 (2011)

Cachinero, J. A.; Análisis y modelado multicast interdominio para el soporte de servicios de video. Madrid (España): Universidad Técnica de Madrid (2009)

Chae Young Lee, Chang Ki Hong y Kang Yong Lee; Reducing Channel Zapping Time in IPTV Based on User's Channel Selection Behaviors, IEEE Transactions on Broadcasting, 56(3), 321-330 (2010)

Cho C., Han I., Jun Y. y Lee H.; Improvement of Channel Zapping Time in IPTV Services Using the Adjacent Groups Join-Leave Method. The 6th International Conference on Advanced Communication Technology, 971-975 (2004)

Cuadra, A.; Supervisión de la calidad percibida en plataformas de IPTV. XVII Jornadas Telecom I+D(0-0). Valencia: Universidad Politécnica de Valencia (2007)

Cuéllar, Juan C., Ortiz, Jesús H. y Arciniegas, José L.; Clasificación y Análisis de Métodos para medir Calidad de la Experiencia del Servicio de Televisión sobre Protocolo IP (IPTV), doi: 10.4067/S071807642014000500017, Inf. Tecnol. (en línea), 25(5), 121-128 (2014).

Díaz, A. y Merino, P.; Un estudio práctico del rendimiento del servicio de Streaming de Video sobre redes móviles GPRS/UMTS. Málaga (España): Universidad De Málaga (2010)

Diez, J. M. y Casares, V.; Modelo de Tráfico para Vídeo MPEG VBR Escalable y no Escalable, IEEE Latin America Transactions, 3(3) (2005)

Eunji L., Jung K. y Hyokyung B.; An efficient hot channel identification scheme for IPTV channel navigation, IEEE Transactions on Consumer Electronics, 60(1), 124-129 (2014)

Ferro, R. A. y C. Hernández; Los sistemas IPTV ¿una amenaza inminente para los actuales medios de teledifusión?, Revista Tecnura, 15(28), 101-122 (2011)

Garrett, M. W. y Willinger W.; Analysis; Modeling and Generation of Self-Similar VBR Video Traffic. Londres: Proceedings de ACM Sigcomm'94, 269-280 (1994)

Joskowicz, J. y Sotelo, R.; Medida de la calidad de voz en redes IP Montevideo (Uruguay): IIE/FiNG/UDELAR, FI/UM (2005)

Lloret J. y Garcia M.; A QoE management system to improve the IPTV network, Int. J. Commun. Syst, 24, 118-138 (2011)

Lundqvist Thomas y Cedervall Mats; Method and Server for Fast Channel Change in Unicast-Multicast IPTV Networks, US 20130332974 A1, PCT/SE2011/050082 (2011)

Manzato D. A. G. y Da Fonseca N. L. S.; A survey of channel switching schemes for IPTV, IEEE Communications Magazine, 51(8), 120-127 (2013)

Martelo R. J., Orozco R. Y. y Puello P.; Guía de Aplicaciones para Implementar Televisión por Protocolo de Internet (IPTV) en las Organizaciones de Cartagena, Colombia. doi: 10.4067/S0718-07642015000400013, Inf. Tecnol. (en línea), 26(4), 97-104 (2015) 
Moumtadi F., Escobar-Argota M., López-Moreno R. y Landeros-Ayala S.; Reducción del retardo en el cambio de canal en servicios IPTV. México: UNAM (2008)

Orbe, C.; Estudios de migración de sistemas de audio y video por suscripción bajo la modalidad de cable físico a IPTV con sugerencias en el ámbito regulador. Quito: Escuela Politécnica Nacional (2010)

Pagan G., Mata J. y Salient S.; Análisis y modelado del tráfico agregado de video MPEG4 en redes MTA. Barcelona (España): Depto de Matemática Aplicada y Tática, Universidad Politécnica de Cataluña (1995)

Ramos F. M. V.; Mitigating IPTV zapping delay, IEEE Communications Magazine, 51(8), 128-133 (2013)

Ramos F., Song F., Rodriguez P., Gibbens R., Crowcroft J., y White I. H.; Constructing an IPTV Workload Model, SIGCOMM, Barcelona, Spain, august (2009)

Sánchez, E.; Implementación de IPTV a través de enlaces de internet de banda ancha (Televisión sobre IP). En Tesis, Guatemala: Universidad de San Carlos de Guatemala, 1-272 (2008)

Santos, A.; Estado del arte en IPTV, Multimedia e Internet, España: Universidad de Vigo, 1-8 (2009)

Song, J., T. Jang y S. Y. Sohn; Conjoint analysis for IPTV service, Expert Systems with Applications, 36(4), 7860-7864 (2009)

Tanwir S. y Perros H.; A Survey of VBR Video Traffic Models, IEEE Communications Surveys \& Tutorials, 15(4), 1778-1802 (2013)

Vega, J. y Sabogal, G.; Análisis de trazas de video MPEG-4, Bogotá: Fundación Universitaria Manuela Beltrán (2009)

Velásquez, J.; Estudio de una red IP/MPLS para agregar servicios de televisión IP en operadoras telefónicas fijas tradicionales para usuarios residenciales mediante tecnologías XDSL para ciudad de Quito. En Tesis, Quito: Escuela Politécnica Nacional, 1-115 (2010)

Video Services Forum; Recommended Video over IP Metrics, en Video Services Forum, Inc. (VSF). Test and Measurements Activity Group (2006)

Viloria C., Freja J. y Donoso Y.; Análisis de rendimiento de la transmisión de IPTV sobre ADSL, WiFi y LAN Extended, Ingeniería y Desarrollo, 23, 84-103 (2008) 